\title{
LA COMERCIALIZACIÓN Y SU INCIDENCIA EN LA COMPETITIVIDAD DE LA MICROEMPRESA DEL SECTOR DE MUEBLES DE MADERA DEL CANTÓN CUENCA EN LA PROVINCIA DEL AZUAY
}

\author{
THE COMMERCIALIZATION AND ITS IMPACT ON THE \\ COMPETITIVENESS OF THE MICRO FIRMS OF THE WOOD \\ FURNITURE SECTOR OF THE CANTON CUENCA IN THE PROVINCE \\ OF AZUAY
}

\author{
Diego Marcelo Cordero Guzmán ${ }^{1}$ \\ Katina Vanessa Bermeo Pazmiño²
}

1 Unidad Académica de Tecnologías de la Información y la Comunicación. Universidad Católica de Cuenca (UCACUE). Cuenca, Ecuador. dcordero@ucacue.edu.ec

2 Unidad Académica de Administración. Universidad Católica de Cuenca (UCACUE). Ecuador.

\section{RESUMEN}

En el presente trabajo se realiza el análisis del proceso de comercialización en la competitividad de la microempresa del sector de fabricación de muebles de madera del cantón Cuenca en la provincia del Azuay en la República del Ecuador. Para ello, se propone el modelo de análisis de competitividad, el mismo que está formado por cuatro constructos o variables. La primera variable denominada "Merc_Ventas", se relaciona con las técnicas de mercadeo y ventas, se compone de diez indicadores, entre los que se encuentran: plan de mercadeo, mercado objetivo, segmentos de mercado, cuotas de venta, información de la competencia entre otros. La segunda variable denominada "Servicios", con sus tres indicadores: responsabilidad del servicio, nivel de satisfacción del cliente y catálogos de servicios. La tercera variable nominada "Distribución", con sus tres indicadores: fuerza de ventas, sistemas de distribución y estructura de vendedores. Y la última variable que mide el nivel de competitividad alcanzado. El trabajo inicia con el análisis de la literatura, de donde surgen las preguntas de investigación; luego se plantean las hipótesis a ser demostradas, se continúa con la validación y comprobación del modelo a través de ecuaciones estructurales; al final se analizan los resultados, las conclusiones y futuras investigaciones.

Palabras Claves: Mercadeo y Ventas, Servicios, Distribución, Competitividad

\begin{abstract}
The present article analyzes the commercialization process on the competitiveness of the micro firms of the wood furniture manufacturing sector at canton Cuenca in the province of Azuay in the Republic of Ecuador. To this end, the analysis of a competitiveness model is proposed, the same one that is formed by four constructs or variables. The first variable called "Merc_Ventas", is related to marketing and sales techniques, is composed of ten indicators, among them are: marketing plan, target market, market segments, sales quotas, competition information among others. The second variable called "Services", has three indicators: service responsibility, level of customer satisfaction and service catalogs. The third variable named "Distribution", has three indicators: sales force, distribution systems and vendor structure. And the last variable that measures the level of competitiveness achieved. The article begins with the analysis of the literature, from where the research questions are established; then the hypotheses to be demonstrated, the validation and verification of the model continues through structural equations; at the end, the results, conclusions and future researches are analyzed.
\end{abstract}

Keywords: Marketing and Sales, Services, Distribution, Competitiveness.

Recibido: 16 de octubre de 2017 Aprobado: 05 de febrero de 2018

Publicado: 20 de junio de 2018 


\section{1.- Introducción}

La competitividad marca la diferencia entre las organizaciones que interactúan en razón de la región, el tipo de organización, el mercado, los productos y servicios; dependiendo del punto de referencia del análisis (Piñeiro, 1993). Es notorio que la evolución de la competitividad en América Latina y el Caribe se ha concentrado en pocos países, sectores y empresas; y en cuatro áreas de acción importantes: fortalecer la atracción de la inversión extranjera directa, vincular empresas líderes con las restantes, articular redes globales de conocimiento y crear alianzas estratégicas con competidores globales (1).

La competitividad internacional relacionada con la estrategia empresarial y el papel de las regiones define tres temas conexos: el contexto internacional cada vez más abierto y globalizado, la estrategia competitiva de los agentes internacionales que organiza la actividad productiva y logra incrementos de productividad en las empresas, y el papel que desempeñan las regiones como creadoras del entorno y estimuladores de ventajas competitivas dinámicas (2).

Los factores de éxito para la competitividad de la PYME son claramente identificados, en donde destacan: gestión de recursos humanos, capacidades directivas de marketing, calidad, innovación, recursos tecnológicos, sistemas de información, gestión financiera, valores culturales, estructura organizativa y know how (3).

En el caso del Ecuador, no se encuentran expuestos con precisión los índices de competitividad en las empresas, motivo por el cual es imprescindible realizar investigaciones relacionadas con el tema, más todavía si un 95\% de la dinámica comercial está cubierto por la actividad de la micro, pequeña y mediana empresa (4).

La presente investigación toma como referencia base: primero, el Mapa de Competitividad del BID, que considera ocho áreas organizacionales consideradas críticas en importancia, que son motores para generar competitividad, y son: planeación estratégica, producción y operaciones, aseguramiento de la calidad, comercialización, contabilidad y finanzas, recursos humanos, gestión ambiental, sistemas de información; y en segundo lugar el instrumento de medición adaptado, diseñado y validado por Saavedra García, (5), basado en el Mapa de Competitividad, del cual se toma para análisis la comercialización y su incidencia en la competitividad.
El área de comercialización, abarca tres variables: mercadeo y ventas, que se compone de diez indicadores; servicios, con tres indicadores; $y$ distribución, con tres. Con estas tres variables y la competitividad, se elabora el modelo para análisis de competitividad para la microempresa del sector de fabricación de muebles de madera.

En el modelo se identifican tres hipótesis, a ser probadas con la investigación, las mismas que constituyen relación causal entre variables, conformando un modelo de ecuaciones estructurales, el mismo que será probado estadísticamente a través de la técnica PLS (Partial Least Square), de utilidad en investigaciones administrativas (6).

El modelo es sujeto de prueba, con datos levantados en 199 microempresas dedicadas a la fabricación de muebles de madera en el cantón Cuenca en la provincia del Azuay, en la República del Ecuador, el instrumento aplicado es de tipo encuesta con 17 preguntas 0 indicadores, sobre comercialización.

Los datos del modelo son testeados a través de estadísticos de ecuaciones estructurales haciendo para ello uso de la herramienta de software SMART PLS 3.1.9. En los resultados obtenidos, se valida que, para este sector microempresarial, el Mercadeo y Ventas influyen de manera positiva sobre la competitividad; no sucediendo lo mismo con los servicios y la distribución. Variables que tendrán que ser analizadas con mayor detalle en futuras investigaciones.

\section{Materiales y Métodos}

\subsection{Marco Teórico}

A continuación en el análisis de la literatura, se explota en detalle las variables analizadas: mercadeo y ventas, servicios, distribución y competitividad.

\section{Mercadeo y Ventas}

La planeación de la comercialización dentro de las MIPYMES incorpora una seria de estrategias y tácticas, tales como la reducción de costos, la mejora de rendimientos y productividad, y el mercadeo cuantitativo (7).

La efectividad del mercadeo en las pequeñas y medianas empresas depende de una mezcla de variables, entre las que se identifican: administrativas, del microentorno, del macroentorno y operativas. Se prueba que existe una relación significativa entre las variables de tipo operativas vs. la efectividad del mercado, y que el tamaño de la empresa 
tiene influencia directa sobre el resto de las variables mencionadas (8).

El mercadeo y ventas involucra los productos y servicios de una empresa que deben distribuirse, formando parte fundamental del marketing mix, pues identifica variables comerciales bajo el control empresarial, y que influencia notoriamente en las estrategias de comercialización con las que cuente la empresa, en las características del producto y en el precio final de venta (9). Las acciones de mercado que llevan adelante las empresas impactan en la sociedad, y es allí en donde se concretizan las relaciones de intercambio exitosas dentro del macro y micro ambiente (10).

El vendedor es considerado como pieza clave de la fuerza de ventas, pues al realizar su labor contribuye a aumentar las utilidades y a obtener altos índices de satisfacción del cliente, a la vez se convierte en el enlace entre proveedor del producto y el cliente que lo conecta (11).

Las ventas se convierten en un eslabón decisivo entre la empresa y los clientes, a través de la transferencia de información de negocios hacia el cliente (material publicitario, promociones, entre otros) y al mismo tiempo defendiendo los intereses de la empresa a la que pertenece (12).

En esta parte del análisis de la literatura, se plantea la pregunta de investigación: ¿Cómo influye el mercadeo y ventas sobre la competitividad de la microempresa de fabricación de muebles de madera?

\section{Servicios}

Los productos que las empresas ofrecen responden a las necesidades de sus clientes y se considera a la innovación como una variable clave en dichos productos; consiste en la utilización productiva de algo nuevo: bienes con calidad, método productivo, mercadeo, fuentes de materias primas 0 esquemas de organización (Berumen, 2008). Los estudios sobre innovación en productos principalmente se han centrado en empresas grandes (sin dejar de lado a las pequeñas empresas), que por su estructura organizacional y su carácter de orientación al mercado, son por naturaleza innovadoras (13).

La satisfacción del cliente hacia los productos y servicios de la empresa debe ser analizada adecuadamente, y el reto está en que la MIPYME logre aumentar el valor de sus productos y servicios incorporando valorconocimiento a la totalidad de la organización. Los conceptos relativos a la innovación como actividad primordial e integradora dentro de una empresa competitiva son claves para alcanzar el objetivo (14).

Las variables compuestas para los factores de competitividad: tecnologías de información, innovación, esfuerzos comerciales, recursos humanos, calidad y tecnología, con la variable ventas como elemento dependiente, da como resultado que las variables de recursos humanos y calidad tengan un peso reducido, y no resulta más importante la innovación que los esfuerzos comerciales, sino que estas dos variables crean sinergia y se refleja en el concepto de ventas (15).

La gestión del talento humano dentro de las empresas marca un viraje en dirección al cliente sea éste interno o externo; tanto gerentes y empleados orientan acciones a la satisfacción del cliente. El entrenamiento en calidad y productividad es intensivo, obligatorio y cíclico, hacia la competitividad. La satisfacción del cliente cede lugar al esfuerzo impecable de atraer al cliente y sobrepasar sus expectativas (16).

Así la pregunta planteada: ¿Cómo influyen los servicios sobre la competitividad de la microempresa de fabricación de muebles de madera?

\section{Distribución}

La distribución considerada como una función clave para las empresas está dentro del concepto de logística que da a los negocios reglas para el control adecuado de los elementos de aprovisionamiento y distribución que impactan en la satisfacción del cliente, y en sus costos y beneficios (17).

La gerencia de ventas necesita la información adecuada por parte de sus vendedores, por la retroalimentación que ellos reciben por parte de sus clientes, para controlar de forma apropiada la fuerza de ventas de la empresa. Es importante tanto la información como los resultados que se obtienen de la correcta realización de la labor de ventas (18).

La capacitación de los vendedores (fuerza de ventas) constituye adiestrar, distribuir el tiempo de los vendedores en las actividades que se le asignen, mejorar el servicio a los clientes a través de presentaciones claras de productos y servicios; mantener comunicación perma-nente con vendedores sobre sus objetivos de ventas, entre otras actividades que se relacionan directamente como la estrategia de competitividad de la empresa (19).

De acuerdo a lo anotado, ¿Cómo influye la distribución sobre la competitividad de la 
microempresa de fabricación de muebles de madera?

\section{Competitividad}

La fuerza de ventas y la competitividad es una relación necesaria, considerando a la competitividad como la capacidad de las empresas para mostrarse al exterior y a la vez logrando una eficiencia interna de sus procesos, productos y personal; y que a partir de dicha eficiencia se consiguen las ganancias y la rentabilidad, requisitos indispensables para perdurar en el tiempo (20).

La globalización hace que las empresas reinventen sus estrategias, pues cada vez se hace más difícil competir en los mercados domésticos e internacionales. Por esto, la clave está en potenciar activos y procesos propios de su cadena de valor para que logren ventajas competitivas y llevan a la empresa a seleccionar las oportunidades, todavía por explotar, para las que está mejor dotada (21).

La competitividad podría definirse como la capacidad relativa de las empresas de un país para producir y comercializar productos de una calidad superior a precios más bajos. De esta forma el concepto de competitividad de una nación ha ido evolucionando relacionada con el entorno local, siendo sus determinantes los factores endógenos de la propia economía nacional (22).

La competitividad relacionada con las ventajas comparativas derivadas de sus recursos, ya sea tierra, fuerza laboral y capital, o con las ventajas creadas derivadas principalmente de la inversión en formación de capital humano y en esfuerzos de innovación, toma en cuenta las áreas internas de la empresa (comercialización), con el objetivo de fortalecer la habilidad para operar rentablemente en un mercado determinado (23).

El presente artículo toma a la comercialización como elemento importante de análisis para mejorar los niveles de competitividad de las MIPYMES.

\section{Metodología}

El problema radica en concebir el modelo para análisis de competitividad, en función de los constructos: mercadeo y ventas, servicios, distribución y competitividad.

A partir de las preguntas de investigación se plantean tres hipótesis, como se especifica a continuación:

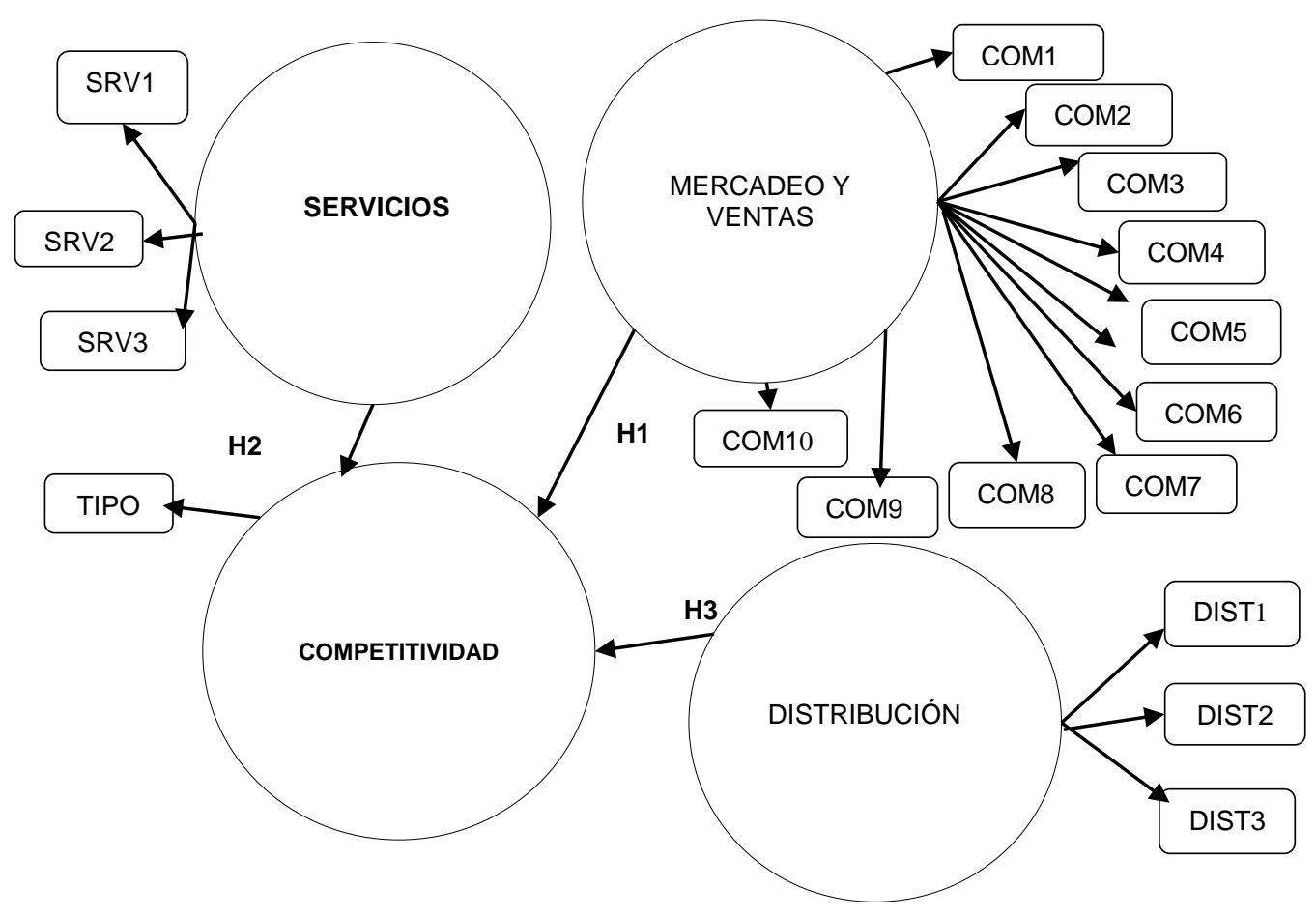

Figura 1. Modelo para análisis de Competitividad en función de la Comercialización

H1: El mercadeo y ventas influyen de manera positiva sobre la competitividad de la microempresa de fabricación de muebles de madera.
H2: Los servicios influyen de manera positiva sobre la competitividad de la microempresa de fabricación de muebles de madera.

H3: La distribución influye de manera positiva sobre la competitividad de la microempresa de fabricación de muebles de madera. 


\section{Modelo propuesto}

Los modelos de ecuaciones estructurales al ser usados en investigaciones administrativas, permiten resolver relaciones causales entre variables(24). En la investigación se hace uso de la técnica PLS (Partial Least Square), o mínimos cuadrados parciales, que evalúa dos componentes, el modelo de medida y el estructural (25).

Con las hipótesis planteadas, se propone el modelo especificado en la figura 1, en donde constan cuatro constructos: Mercadeo y Ventas; Servicios; Distribución; y Competitividad.

Mercadeo y Ventas contiene diez indicadores, que van desde COM1 a COM10; servicios con tres indicadores desde SRV1 hasta SRV3; distribución, con tres indicadores, de DIST1 hasta DIST3; y Competitividad, con un solo indicador denominado TIPO; en total se contabilizan 17 indicadores.
En base a la teoría, de acuerdo a las características del modelo estructural, en función del número de constructos que apuntan a una variable dependiente, se puede determinar el tamaño de la muestra, multiplicando este número por 10; en el presente trabajo se toma esta recomendación, a pesar de que existen diferentes perspectivas para encontrar el tamaño ideal en función del número de constructos e indicadores por constructo (26). Con este antecedente para el modelo en cuestión, son suficientes 30 encuestas para probar el modelo, debido a que tres variables: servicios, mercadeo y ventas, distribución apuntan hacia competitividad. A pesar de ello para disponer de altos niveles de consistencia se recabaron 199 encuestas.

\section{Instrumento para colección de información}

Para recabar datos se ha diseñado un instrumento (tipo encuesta) con 17 preguntas como se indica en la Tabla No.1.

\section{Población y muestra}

Tabla 1. Instrumento para captura de datos

\begin{tabular}{|c|c|c|}
\hline ID & Items & Escala \\
\hline \multicolumn{3}{|c|}{ 1. MERCADEO Y VENTAS } \\
\hline COM1 & $\begin{array}{l}\text { ¿El proceso de planeación genera un plan de mercadeo anual, escrito y detallado, con } \\
\text { responsables e índices de gestión claramente definidos? }\end{array}$ & 012345 \\
\hline COM2 & $\begin{array}{l}\text { ¿La empresa tiene claramente definido su mercado objetivo, sus estrategias de } \\
\text { penetración, posicionamiento y comercialización? }\end{array}$ & 0112345 \\
\hline COM3 & $\begin{array}{l}\text { ¿La empresa conoce los segmentos del mercado en que compite, su participación, } \\
\text { crecimiento y rentabilidad y desarrolla estrategias comerciales escritas para cada uno de } \\
\text { ellos? }\end{array}$ & 0123345 \\
\hline COM4 & $\begin{array}{l}\text { ¿La empresa establece objetivos o cuotas de venta, de recaudo y de consecución de } \\
\text { clientes nuevos a cada uno de sus vendedores y controla su cumplimiento? }\end{array}$ & 012345 \\
\hline COM5 & $\begin{array}{l}\text { ¿La empresa dispone de información de sus competidores (en cuanto a reputación, } \\
\text { calidad de sus productos y servicios, fuerza de ventas y precios)? }\end{array}$ & 0112345 \\
\hline COM6 & $\begin{array}{l}\text { ¿Las estrategias, objetivos y precios de la empresa están determinados con base en } \\
\text { sus costos, la oferta, la demanda y la situación competitiva? }\end{array}$ & 012345 \\
\hline COM7 & $\begin{array}{l}\text { ¿En los últimos dos años, los productos nuevos (menores de } 3 \text { años) han generado un } \\
\text { porcentaje importante de las ventas y de las utilidades totales de la empresa? }\end{array}$ & 012345 \\
\hline COM8 & $\begin{array}{l}\text { Los recursos asignados al marketing (material publicitario, promociones, etc.) son } \\
\text { adecuados y se usan de manera eficiente? }\end{array}$ & 012345 \\
\hline COM9 & $\begin{array}{l}\text { ¿La empresa dispone de un sistema de información y análisis que le permite obtener } \\
\text { información actualizada sobre sus clientes, sus necesidades y los factores que guían } \\
\text { sus decisiones de compra? }\end{array}$ & 012345 \\
\hline COM10 & $\begin{array}{l}\text { ¿La empresa evalúa periódicamente sus mecanismos de promoción, sistemas de } \\
\text { información de mercados y seguimiento de tendencias? }\end{array}$ & 012345 \\
\hline 2 & SERVICIOS & \\
\hline SRV1 & $\begin{array}{l}\text { El personal que tiene contacto con el cliente es consciente de sus responsabilidades y } \\
\text { tiene suficiente autonomía para atender adecuadamente sus necesidades. }\end{array}$ & 0122345 \\
\hline SRV2 & $\begin{array}{l}\text { La empresa tiene un sistema de investigación que le permita conocer el nivel de } \\
\text { satisfacción del cliente, lo documenta y toma acciones con base en su análisis. }\end{array}$ & 0122345 \\
\hline SRV3 & La empresa dispone de catálogos y especificaciones técnicas de sus productos. & 01123445 \\
\hline & DISTRIBUCIÓN & \\
\hline DIST1 & $\begin{array}{l}\text { La empresa posee una fuerza de ventas capacitada, motivada y competente que apoya } \\
\text { el cumplimiento de los objetivos de la empresa. }\end{array}$ & 0122345 \\
\hline DIST2 & $\begin{array}{l}\text { La empresa ha desarrollado un sistema eficiente de distribución que permite llevar sus } \\
\text { productos a sus clientes cuando y donde ellos los necesitan. }\end{array}$ & 0122345 \\
\hline DIST3 & $\begin{array}{l}\text { La empresa prefiere contratar vendedores con vínculo laboral en lugar de independientes } \\
\text { sin vínculo laboral. }\end{array}$ & 0122345 \\
\hline 3. & COMPETITIVIDAD & \\
\hline TIPO & ¿El nivel de competitividad actual es ? & 12345 \\
\hline
\end{tabular}


El modelo es analizado a través de dos fases:

Validez y Fiabilidad del Modelo de Medida

En donde se valida si la teoría se soporta con las variables observadas; en la Figura No.2 se

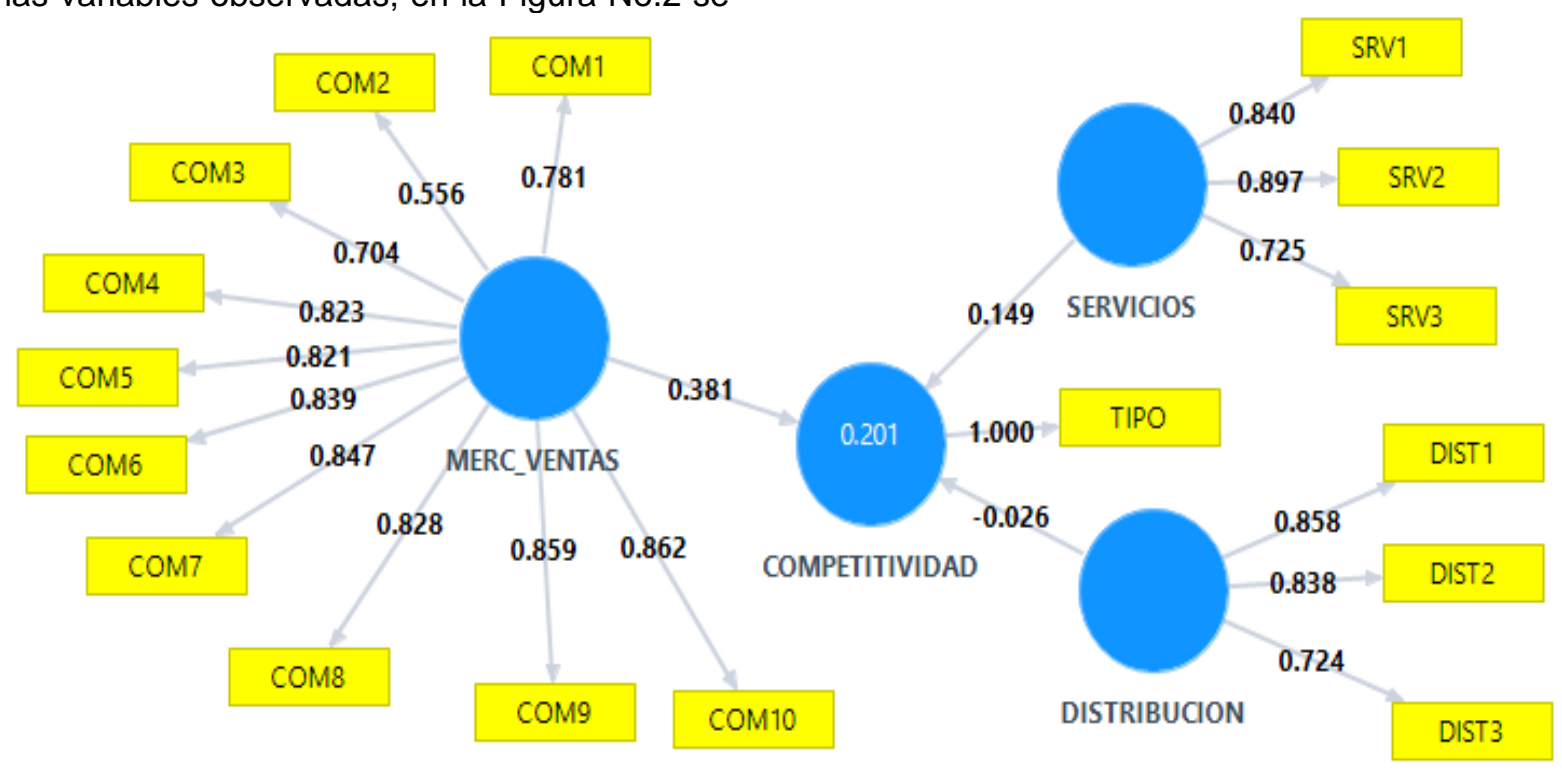

ilustran los resultados del modelo estructural. En la Tabla No.2 se desglosan los parámetros de esta evaluación.

Figura 2. Resultados del modelo estructural

Tabla 2. Fiabilidad del modelo de medida.

\begin{tabular}{|l|l|}
\hline \multicolumn{1}{|c|}{ Parámetros } & \multicolumn{1}{|c|}{ Valores } \\
\hline Fiabilidad individual del ítem. & $\begin{array}{l}\text { Todas las cargas están por encima de 0.7, a excepción del indicador } \\
\text { COM2, lo que implica que este indicador no aporta al constructo. Ver } \\
\text { figura 2, valores entre constructos e indicadores. }\end{array}$ \\
\hline Fiabilidad de cada constructo & $\begin{array}{l}\text { Los valores allfa de Cronbach de los constructos superan el valor 0.7, lo } \\
\text { que les da validez constructo, como se indica en la Tabla 3. } \\
\text { En el análisis de fiabilidad compuesta, todos los constructos del modelo } \\
\text { presentan valores superiores a 0.6, confirmándose por tanto la } \\
\text { consistencia interna de todos ellos como se ilustra en la Tabla 4. }\end{array}$ \\
\hline Validez convergente (AVE) & $\begin{array}{l}\text { Los valores para el indicador AVE, superan el valor mínimo recomendable } \\
\text { de 0.5 Tabla 5. }\end{array}$ \\
\hline Validez discriminante & $\begin{array}{l}\text { Con el chequeo de cargas cruzadas, se valida que cada indicador tiene } \\
\text { correlación con su propia variable latente antes que con otras, como se } \\
\text { indica en la Tabla 6. }\end{array}$ \\
\hline
\end{tabular}

Tabla 3. Fiabilidad del constructo, compuesta y validez convergente

\begin{tabular}{|l|c|c|c|}
\hline & Cronbachs Alpha & Composite Reliability & AVE \\
\hline COMPETITIVIDAD & 1.000 & 1.000 & 1.000 \\
\hline DISTRIBUCION & 0.733 & 0.850 & 0.654 \\
\hline MERC_VENTAS & 0.935 & 0.945 & 0.635 \\
\hline SERVICIOS & 0.765 & 0.863 & 0.679 \\
\hline
\end{tabular}


Tabla. 4. Cargas cruzadas. Validez discriminante

\begin{tabular}{|c|c|c|c|c|}
\hline & COMPETITIVIDAD & DISTRIBUCION & MERC_VENTAS & SERVICIOS \\
\hline COM1 & 0.341 & 0.332 & 0.781 & 0.304 \\
\hline COM10 & 0.410 & 0.345 & 0.862 & 0.320 \\
\hline COM2 & 0.183 & 0.431 & 0.556 & 0.364 \\
\hline COM3 & 0.297 & 0.392 & 0.704 & 0.236 \\
\hline COM4 & 0.354 & 0.370 & 0.823 & 0.330 \\
\hline COM5 & 0.360 & 0.377 & 0.821 & 0.366 \\
\hline COM6 & 0.358 & 0.371 & 0.839 & 0.350 \\
\hline COM7 & 0.332 & 0.370 & 0.847 & 0.313 \\
\hline COM8 & 0.316 & 0.304 & 0.828 & 0.319 \\
\hline COM9 & 0.407 & 0.300 & 0.859 & 0.341 \\
\hline DIST1 & 0.196 & 0.858 & 0.354 & 0.512 \\
\hline DIST2 & 0.147 & 0.838 & 0.403 & 0.501 \\
\hline DIST3 & 0.181 & 0.724 & 0.314 & 0.263 \\
\hline SRV1 & 0.233 & 0.437 & 0.185 & 0.840 \\
\hline SRV2 & 0.287 & 0.451 & 0.506 & 0.897 \\
\hline SRV3 & 0.176 & 0.416 & 0.256 & 0.725 \\
\hline TIPO & 1.000 & 0.220 & 0.430 & 0.289 \\
\hline
\end{tabular}

La valoración del modelo estructural, para medir la consistencia de las relaciones entre las variables y la comprobación de hipótesis. En la Tabla 5, se adjuntan los resultados de la evaluación.

Tabla 5. Evaluación del modelo estructural.

\begin{tabular}{|l|l|}
\hline \multicolumn{1}{|c|}{ Parámetro } & \multicolumn{1}{|c|}{ Valores } \\
\hline Índice $R^{2}$ & $\begin{array}{l}\text { EI modelo es altamente predictivo a través de su variable dependiente } \\
\text { COMPETITIVIDAD, el valor obtenido para }{ }^{2} \text { es superior a 0.1, como se indica } \\
\text { en la Tabla 8. }\end{array}$ \\
\hline Efecto $f^{2}$ & $\begin{array}{l}\text { Mide el impacto sobre la COMPETITIVIDAD de las variables latentes: } \\
\text { MERC_VENTAS, SERVICIOS, DISTRIBUCION. La variable DISTRIBUCIÓN, } \\
\text { no se encuentra en el rango permisible, como se indica en la Tabla 9 }\end{array}$ \\
\hline $\begin{array}{l}\text { Coeficientes path } \\
\text { estandarizados } \beta\end{array}$ & $\begin{array}{l}\text { Para el modelo supera el valor mínimo estándar de 0.2, solamente el camino } \\
\text { entre la variable MERC_VENTAS y COMPETITIVIDAD, como se indica en la } \\
\text { Tabla 10. }\end{array}$ \\
\hline
\end{tabular}

Tabla 6. $R^{2}$ de la variable latente dependiente

\begin{tabular}{|l|c|}
\hline & R Square \\
\hline COMPETITIVIDAD & 0.201 \\
\hline
\end{tabular}

Tabla 7. $f^{2}$ de la variable latente dependiente

\begin{tabular}{|c|c|l|l|l|}
\hline & COMPETITIVIDAD & DISTRIBUCION & MERC_VENTAS & SERVICIOS \\
\hline COMPETITIVIDAD & & & & \\
\hline DISTRIBUCION & 0.001 & & & \\
\hline MERC_VENTAS & 0.140 & & & \\
\hline SERVICIOS & 0.019 & & & \\
\hline
\end{tabular}


Tabla 8. Coeficientes path

\begin{tabular}{|l|r|l|l|l|}
\hline & COMPETITIVIDAD & DISTRIBUCION & MERC_VENTAS & SERVICIOS \\
\hline COMPETITIVIDAD & & & & \\
\hline DISTRIBUCION & -0.026 & & & \\
\hline MERC_VENTAS & 0.381 & & & \\
\hline SERVICIOS & 0.149 & & & \\
\hline
\end{tabular}

En la Tabla 9 constan las relaciones entre los constructos del modelo a través de los caminos beta estandarizados, el error estándar, el valor de $t$ de Student, el nivel de significancia y la aceptación o rechazo de la hipótesis. Para este ámbito los valores "t" de Student se consideran como significativos a aquellos cuyos valores son superiores a 1,96.

Tabla 9. Relaciones entre constructos

\begin{tabular}{|l|c|c|c|c|c|c|}
\hline & $\beta$ & $\begin{array}{c}\text { Error } \\
\text { estándar }\end{array}$ & $\mathrm{t}$ - Student & $\begin{array}{c}\text { Valores } \\
\mathrm{p}\end{array}$ & $\begin{array}{c}\text { Nivel de } \\
\text { significancia }\end{array}$ & $\begin{array}{c}\text { Aceptación } 0 \\
\text { rechazo }\end{array}$ \\
\hline $\begin{array}{l}\text { DISTRIBUCION -> } \\
\text { COMPETITIVIDAD }\end{array}$ & -0.026 & 0.094 & 0.278 & 0.781 & & Rechazado \\
\hline $\begin{array}{l}\text { MERC_VENTAS -> } \\
\text { COMPETITIVIDAD }\end{array}$ & 0.381 & 0.063 & 6.057 & 0.000 & $* \star \star$ & Aceptado \\
\hline $\begin{array}{l}\text { SERVICIOS -> } \\
\text { COMPETITIVIDAD }\end{array}$ & 0.149 & 0.082 & 1.810 & 0.071 & & Rechazado \\
\hline
\end{tabular}

${ }^{* * *} p<0.001 ;{ }^{* *} p<0.01 ;{ }^{*} p<0.05$

\section{Conclusiones}

Con respecto a las hipótesis, se ha encontrado que la H2 manifiesta relación altamente significativa entre la variable Mercadeo y Ventas y la Competitividad. Resultado que se apoya los hallazgos de que la mayoría de micro y pequeños empresarios buscan continuamente la actualización de sus procesos a través de la evolución de sus ventas acompañado del desarrollo impresionante de la tecnología, así mismo las técnicas y estrategias de comercialización dentro de un mercado cambiante y en donde prevalece los gustos y preferencias de los consumidores; el objetivo es conseguir, mantener y lograr la fidelidad del mercado en un reto constante.

Las hipótesis uno y tres son rechazadas, por no cumplir los parámetros mínimos de significancia. Para discernir el ¿por qué?, la distribución y servicios no resultan factores preponderantes para el incremento de la competitividad en las microempresas de muebles de madera, se describen a continuación las posible razones: no existen todavía canales adecuados de distribución, que estén soportados con un manejo técnico y metódico; por otro lado los servicios no están siendo en este tipo de microempresas un valor agregado, para ser más competitivos.

\section{Futuras investigaciones}

Para futuras instancias, se recomienda ahondar en investigaciones de corte mixto para detectar las razones en detalle por las cuales, la falta de adecuados canales de distribución y servicios apropiados afecten de manera negativa a la competitividad en las microempresas de fabricación de muebles de madera en la provincia del Azuay.

\section{Agradecimientos}

A la Universidad Católica de Cuenca, por la apertura a procesos de orden investigativo sobre la temática expuesta.

\section{Referencias}

1. Mortimore M, Perez Nuñez W. La Competitividad Empresarial en América Latina y el Caribe. Revista de la CEPAL. 2001 Agosto;(74): p. 37-59.

2. Alburquerque Llorens F. Competitividad Internacional, Estrategia Empresarial y Papel de las Regiones. In.: Instituto Latinoamericano y del Caribe de Planificación Económica y Social. Naciones Unidas. CEPAL.; 1995.

3. Rubio Bañon A, Aragon Sanchez A. Factores explicativos del éxito competitivo. 
Un estudio empírico en la PYME. Cuadernos de Gestión. 2002; 2(1): p. 49-63.

4. INEC. Avances del Censo Nacional Económico y Mecanismos de Difusión.. Cuenca: Instituto Nacional de Estadísticas y Censos; 2012.

5. Saavedra García ML. Hacia la Determinación de la Competitividad de la PYME Latinoamericana Ciudad de México: Publicaciones Empresariales UNAM. FCA Publishing. ; 2014.

6. Wold H. Model Construction and Evaluation when Theoretical Knowledge Is Scarce: An Example of the Use of Partial Least Squares. Genève: Université de Genève; 1979.

7. Johansson JK, Nonaka I. Implacable: ahora sí, por fin, la manera japonesa de hacer marketing.; 1997.

8. Zapata Guerrero EE. La efectividad del mercadeo en las pequeñas y medianas empresas (PYMES) de los sectores industrial y de servicios del departamento de Boyacá, Colombia.. Revista Colombiana de Marketing. 2001; 2(3).

9. Diez de Velasco Vallejo M. Las Organizaciones Internacionales Madrid: Tecnos; 2003.

10.Dess G, Lumpkin GT. Strategic Management: Creating Competitive Advantage. Corporate Governance Update. España: McGrawHill//rwin; 2003.

11. Guiltinan JP. Gerencia de Marketing. 6th ed. Colombia: McGraw Hill; 2005.

12. Kotler $\mathrm{P}$, Armstrong G. Fundamentos de Marketing. Octava Edición ed. México; 2008.

13. Athanasios H. A Resource-based view of innovativeness in small firms. Technology Analysis \& Strategic Management. 2000; 12 : p. 263-281.

14.Castellanos JG. PYMES Innovadoras. Cambio de Estrategias e Instrumentos. Revista Escuela de Administración de Negocios. 2003 Abril;(47): p. 10-33.
15.Aguilera Enriquez L, Gonzalez Adame M, Rodriguez Camacho R. Estrategias empresariales para la competitividad y el crecimiento de las PYMES. Una evidencia empírica.. Investigación y Ciencia. 2011;(53): p. 39-48.

16. Chiavenato I, Villamizar G. Gestion del Talento Humano; 2009.

17.I COS JP. Manual de Logística Integral Madrid: Ediciones Díaz de Santos; 1998.

18.Pride, Ferrell OC. Marketing Concepts and Strategies Boston: Houghton Mifflin; 2000.

19.Fisher L, Espejo J. Mercadotecnia México; 2003.

20.Leon Valbuena NI. Fuerza de ventas determinante de la competitividad empresarial. Revista de Ciencias Sociales. 2013; XIX(2): p. 379-389.

21.Saez de Viteri Arranz D. El Potencial Competitivo de la Empresa: Recursos, Capacidades, Rutinas y Procesos de Valor Añadido.. Investigaciones Europeas de Dirección y Economía de la Empresa. 2000; 6(3): p. 71-86.

22.Ezeala-Harrison F. Theory and Policy of International Competitiveness.. 1999.

23.Padilla R. Instrumentos de Medición de la Competitividad. 2006 Septiembre 27-29..

24.Gil M, Alcaraz J, Iniesta A. FACTORES ADMINISTRATIVOS EN EL ÉXITO DE TQM: UN ANÁLISIS RELACIONAL CON ECUACIONES ESTRUCTURALES. CULCyT. 2015;(45): p. 126-138.

25.Cepeda G, Roldán J. Aplicando en la práctica la técnica PLS en la administración de empresas [Applying the PLS technique in practice in business administration]. Sevilla:; 2008.

26. Vargas T, Mora-Esquivel R. Tamaño de la muestra en modelos de ecuaciones estructurales con constructos latentes: Un método práctico. Revista Actualidades Investigativas en Educación. 2017: p. 1-34. 